\title{
3-Iodothyronamine: A High Potency Metabolic Hormone and its Potential for Therapeutic Applications
}

\author{
Michael Rogowski ${ }^{1}$ and Fariba M. Assadi-Porter ${ }^{2,3,4 *}$ \\ ${ }^{1}$ Department of Nutritional Sciences, Texas Tech University, 1301, Akron Ave., Lubbock, Texas, USA \\ ${ }^{2}$ Department of Biochemistry, University of Wisconsin-Madison, 433 Babcock Dr., Madison, WI, 53706, USA \\ ${ }^{3}$ Department of Zoology, University of Wisconsin-Madison, 250 Mills St., Madison, WI, 53706, USA \\ ${ }^{4}$ NMR Facility at University of Wisconsin-Madison, 433 Babcock Dr., Madison, WI, 53706, USA
}

\begin{abstract}
Received: November 13, 2014; Accepted: February 24, 2015; Published: March 05, 2015
*Corresponding author: Fariba Assadi-Porter, Department of Zoology, University of Wisconsin-Madison, USA, Tel: 608-262-0029; E-mail: fariba@ nmrfam.wisc.edu
\end{abstract}

\begin{abstract}
3-Iodothyronamide (T1AM) is a naturally produced endogenous hormone like molecule. Only recently it has been discovered in the past decade, the proceeding body of research emerging from its initial discovery has revealed a substantial capacity of T1AM as a new potential hormone that affects numerous physiological processes and organs. Initially, it was hypothesized to be a byproduct of Thyroid Hormone $(\mathrm{TH})$ metabolism; however, the current body of evidence suggests that its production and physiological function appear to be uncoupled and dramatically divergent from that of TH. This review summarizes the physiological and biochemical effects of T1AM reported in the literature along with its proposed mechanisms of action and production pathways. The physiological effects of T1AM appear to be dose specific, in some cases exerting opposing effects in the same biological processes. Uptake, storage, and degree of effect appear to be tissue specific as well. From the current body of literature, potential therapeutic applications with T1AM are quite apparent, ranging from sleep/torpidity induction, conferring protection against ischemic injury, and anti-obesogenic by inducing increased metabolic reliance on lipid oxidation. Future research is needed to understand the specific mechanisms of its dose dependent and tissue dependent effects, its mechanism of entry into the cell, its cellular targets, and primary site of production.
\end{abstract}

\section{Introduction}

3-iodothyronamine (T1AM) is an endogenous metabolite and structural analog of Thyroid Hormone (TH). Initially discovered in 2004 by Scanlan et al. [1]. T1AM has become a research area of intense interest due to its rapid induction of hypometabolic effects, largely in opposition to those of $\mathrm{TH}$, and its strong potential for therapeutic applications [2-4]. This recent discovery has opened up a new realm of thyroid metabolism research and continues to broaden in scope to encompass a diverse array of physiologic research fields including cardiovascular [5], neurological [6], cellular redox [4], lipid and carbohydrate metabolism [3], sleep [7], and torpidity [8]. Though other thyronamines have been characterized that can produce similar effects, T1AM remains the most promising thyronamine in physiological research due to its greater potency and receptor affinity than other thyronamines [1].

\section{Production and regulation}

Due to their structural similarities, it was initially hypothesized that T1AM was derived from thyroxine (T4), accomplished through a series of one decarboxylation and three deiodination enzymatic reactions [9]. This conversion of T4 or Triiodothyronine (T3) into T1AM has not been directly demonstrated (Figure 1). It is still an attractive hypothesis for a number of reasons. Decarboxylation and deiodination are known to play an important role in thyroid hormone catabolism [10]. Thyronamines are also able to serve as substrates for deiodinases [11]. This reaction series could explain a functional role of reverse Triiodothyronine (rT3) as an intermediate product of T1AM synthesis, as rT3 is the inactive deiodination product of T4 with no known physiological function, yet it is produced in equal ratios to the active compound T3 [12]. The essential decarboxylation reaction however has not been biologically characterized, as synthesis of thyronamines from $\mathrm{T} 4$ or $\mathrm{T} 3$ would require decarboxylation to occur prior to deamination, as the amine group is retained in thyronamines. This reaction order is not usually thought to occur in thyroid hormone catabolism, but the enzyme that catalyzes this reaction, named aromatic amino acid decarboxylase, is widely expressed and possesses a broad range of substrate specificity. Though this series of reactions seems highly plausible, a more recent study using heavy isotope tracing of exogenous T4 in a mouse model of induced hypothyroidism did not demonstrate any conversion of T4 to T1AM [13]. As a result of induced hypothyroidism, T1AM levels were greatly diminished as well, but exogenous $\mathrm{T} 4$ administration was not able to replenish serum T1AM levels despite successfully restoring circulating T4 and T3 concentrations. This suggests that production of T1AM uses the same biosynthetic factors required for the synthesis of $\mathrm{T} 4$ and provides with the possibility that, T1AM is synthesized de novo in a divergent manner from that of $\mathrm{T} 4$. 
The relationship between $\mathrm{T} 4$ and T1AM production is not clear. Thyroid cancer patients receiving TSH-suppressive LT4 therapy had significantly higher T1AM levels than healthy controls, but no correlation was observed between T1AM and either T4 and TSH serum levels [14]. Due to surgery, radiotherapy, or a combination of both, these patients no longer possessed any functional thyroid tissue, but their T1AM levels remained intact, suggesting an extra thyroid origin of T1AM production. This lack of relation between circulating levels of the two thyroid metabolites was also observed in the same study with a separate cohort of T4 substituted patients with pituitary insufficiency. T1AM levels were not altered following subsequent decrease in T4 levels due to supplement withdrawal in these patients [14]. These findings seem contrast to Hachenmueller et al. [13] mouse model of hypothyroidism which resulted in decreased T1AM levels. This may be explained by the divergent mechanisms of thyroid insufficiency in these study populations. The model of induced hypothyroidism in mice was accomplished by supplementing the animal's drinking water with $0.1 \%$ methimazole and $0.2 \% \mathrm{KClO}_{4}$; these chemicals function to inhibit both the enzyme thyroperoxidase, which catalyzes the addition of the iodine molecule to the thyroglobin molecule, and by inhibiting the function of the $\mathrm{Na}^{+} / \mathrm{I}^{-}$transporter, respectively. This chemical method of induced hypothyroidism would produce a systematic inhibition of thyroperoxidase and $\mathrm{Na}^{+} / \mathrm{I}^{-}$transporters, extending to extra-thyroid tissues, whereas surgery and radiotherapy for thyroid cancer treatment would be limited to the thyroid tissue itself. Taken together, both these findings support the conclusion that the $\mathrm{T} 4$ and T1AM require the same biosynthetic factors and that T1AM's production may be largely in extra thyroid tissues.

This raises questions as to where the major production sites of T1AM are in the body. Expression of thyroperoxidase and $\mathrm{Na}^{+} / \mathrm{I}^{-}$transporters has not been extensively studied or thought to be physiologically relevant to key biosynthetic pathways in extra thyroid tissues. In vitro experiments have shown T1AM production in $\mathrm{H} 9 \mathrm{C} 2$ cardiomyocytes in response to $\mathrm{T} 3$ incubation, but this production was very low [15]. This same study failed to demonstrate production of T1AM in situ with perfused rat hearts incubated with T4 [15]. Without the exact knowledge of how and where it is produced, coupled with an apparent lack of relationship between T1AM circulating thyroid hormone levels [14], it is not clear how the production and secretion of T1AM is regulated or from which tissues it is produced in. To date, there has not been any endogenous compounds identified that demonstrate the ability to increase T1AM levels nor it is known whether chronic T1AM supplementation decreases endogenous production.

Deactivation and degradation of T1AM have been speculated through different mechanisms. Thyronamines have been identified as substrates for Sulfotransferases (SULTs) [16], which are able to catalyze the sulfation of T3 as well. SULTs are phase II

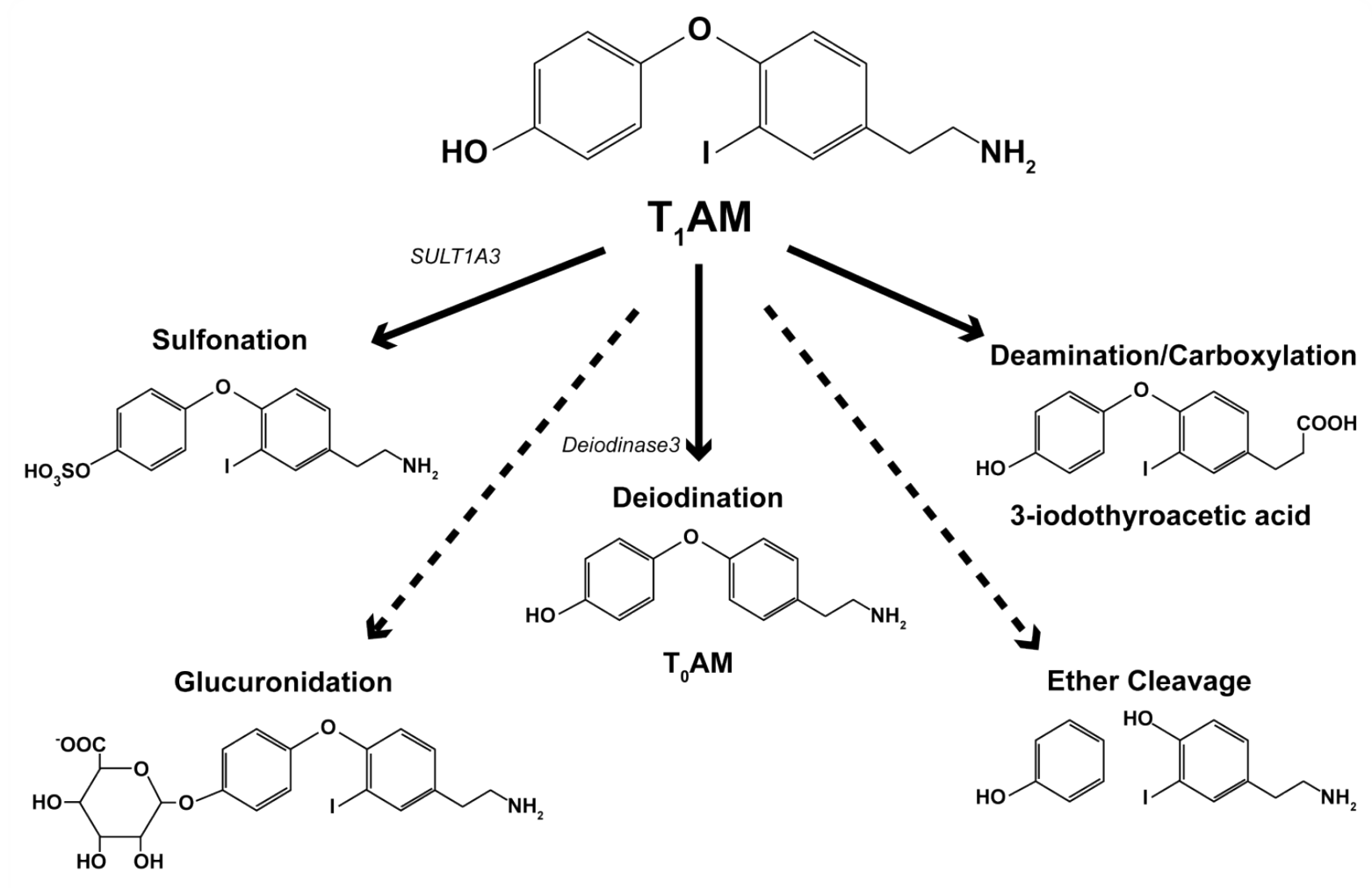

Figure 1: Proposed degradation pathways of T1AM. Solid arrows indicate degradation product formation demonstrated in vitro. Dashed arrows indicate proposed degradation pathways yet to be demonstrated. 
drug-metabolizing enzymes and are thought to play a role in the attenuation and disposal of T1AM. T1AM has been demonstrated to induce greater activity of human liver sulfotransferases than other thyronamines, with SULT-1A3 identified as the primary sulfation enzyme of T1AM [16]. More recently, formation of 3-iodothyroacetic acid (TA1), a deaminated analog of T1AM, has been observed in liver after T1AM administration [17]. This is speculated to be mediated through the action of amine oxidases that convert the amine group of T1AM into an aldehyde, which is then subsequently further oxidized into a carboxylic acid to form TA1. Increased production of TA1 was observed in H9C2 cardio myocytes and perfused rat hearts incubated with T1AM [15]. This conversion was inhibited by iproniazid, a non-specific amine oxidase inhibitor, providing strong evidence for the role of amine oxidases in this catabolic process [15]. It has also been speculated that ether bond cleavage and glucuronidation are additional metabolic breakdown pathways of thyronamines [16], which are known processes in T3 catabolism [10], but have not been explored or demonstrated in the literature at the time of this review (Figure 1).

T1AM mechanism of action through transmembrane spanning G-protein-coupled trace-amine-associated receptors (TAARs)

Prior to its discovery as an endogenous compound, T1AM was proposed to be a potential activator of thyramine receptor, TAAR1. This hypothesis was correct and T1AM was shown to be a more potent activator of TAAR1 compared to other thyronamines [1]. TAARs represent a family of 15 different subtypes that are $G$ protein-coupled receptors (GCPRs) [18]. Whether this activity of T1AM with TAARs interaction is coupled to $G_{s}$ or $G_{i}$ signaling in vivo is not known or if one activation pathway is consistent across different tissues and TAAR subtypes. Initially, T1AM was shown to be able to increase cellular cAMP in response to TAAR1 activation when overexpressed in vitro [1]. This was demonstrated using stable expression of rat and mouse TAAR1 model in HEK cells where cAMP release was shown to be dose dependent with T1AM. However, TAAR1 and other TAARs are not necessarily coupled to the cAMP activation pathway in vivo. TAAR1 knockout mice maintain the dose dependent hypothermic response of T1AM injection of wild type mice [19]. In a later study, using an in situ model of perfused rat heart, T1AM infusion did not induce cAMP production [20]. Other GCPR induction mechanisms were also evaluated, but inhibition of protein kinase A, protein kinase C (PKC), MAP kinase, and CaM-dependent kinase II did not disrupt the negative inotropic response to T1AM in the perfused rat heart. However, T1AM's response was potentiated by treatment with tyrosine kinase and phospholipase C inhibition, while the response to T1AM was blunted with tryrosine phosphatase inhibition [20,21]. These findings suggest that cAMP release is not a primary mediator of T1AM's cellular signaling activation, or is necessary to exert its physiologic effects but rather than that T1AM plays a potentially critical role in the modulation of phosphorylation status of key tyrosine residues in cellular signaling pathways. Whether this response is mediated through a particular TAAR subtype or other receptor remains to be elucidated.
Due to its affinity, TAARs have been the most promising non-genomics mechanism through which T1AM exerts its physiologic effects. Supporting this, structural derivatives of T1AM synthesized to be TAAR1 receptor agonists were also found to induce hypothermia in mice [22]. Unexpectedly a later study demonstrated that, T1AM's potent hypothermic effect was maintained in TAAR1 knockout mice [19]. This suggests that TAAR, or at least TAAR1, agonist activation is not necessary for at least some of the metabolic responses due to T1AM. Radioactive tracing of exogenous ${ }^{125}$ I labeled-T1AM did not indicate any direct relationship with TAAR receptor expression across 8 different TAAR isoforms and measured radioactivity in tissues [23]. Additionally, it was found that expression of TAARs in mice and rat proved to be very low in most tissues; TAAR gene expression was only detected in trace amounts across most tissues, with notable expression levels of TAAR1 in the stomach and testis, and TAAR8 in the testis, intestine, and spleen [23]. However, a later study could not identify any affinity of T1AM with TAAR8 [24]. These findings suggest that T1AM's role in metabolism may extend beyond its ability to interact with TAARs, to possibly yet an alternative intracellular receptor mediated mechanism. Other candidates have been proposed, but TAAR structural homologs dopamine D1 receptor and $\beta 2$ adrenergic receptors were not activated in response to T1AM in vitro [1].

\section{Distribution and transport}

Originally discovered in rat brain [1], T1AM has been detected in a wide panel of tissues [15,23], and is present in plasma $[14,25]$ which is likely the primary means of delivery to target tissues. Distribution of measured radioactivity in response to acute ${ }^{125}$ I labeled-T1AM injection in mice indicated abundant levels of accumulation in stomach, intestine, kidney, and gallbladder within as little as 30 minutes [23]; however, this is thought to reflect excretion pattern of excess T1AM rather than functional activity sites. Elevated radiation levels in liver, fat, and skeletal muscle for a full 24 hours after ${ }^{125}$ I labeledT1AM injection provides evidence for these tissues as possible storage sites of T1AM [23]. Initial detection methods of using LC/ MS revealed very low circulating levels of T1AM around $0.3 \mathrm{nM}$ range [15], however, an anti-body based assay detention method measured circulating T1AM levels in human at approximately 66 nM [14] which approaches levels of circulating T4 (70-150 nM) and those concentrations of T1AM recorded in other tissues [15]. This discrepancy in measured levels is explained by more recent findings that circulating T1AM is largely bound to ApoB-100 containing lipoproteins [25], whereby traditional LC/MS sample extraction methods can exclude protein fractions. This affinity of T1AM binding to ApoB100 was uniquely strong compared to other thyronamines and explains the high abundance of endogenous T1AM in the liver [15]. Measured abundance and half-life duration of T1AM appear to be tissue specific [15,23] but the identity of target tissues responsible for the specific metabolic effects of T1AM remains elusive.

T1AM is believed to primarily mediate its effects through cell surface (GPCRs) interaction, due to its acute rapid onset of metabolic effects [1], but multiple studies have also 
demonstrated cellular uptake of T1AM in vitro $[15,25,26]$ across multiple cell lines, accounting for its ubiquitous distribution in vivo. One study using cultured HepG2 cells treated with [ $\left.{ }^{125} \mathrm{I}\right]$ T1AM revealed that $60 \%$ was membrane associated with $40 \%$ detected in the cytoplasm [23], demonstrating a sizable fraction of T1AM cellular interaction to be internal, in contrast to initial proposed mechanism exclusively through GPCRs. Uptake of T1AM appears to be mediated via facilitated diffusion, but the specific transporters which are responsible for this have yet to be identified [26], however due to its association with ApoB100 in circulation, LDL receptors may likely facilitate for cellular internalization of T1AM [25]. This internalization, and subsequent unknown intracellular interaction, may mediate long term alterations in gene expression patterns, as confirmed by Mariotti et al. in rat adipose and liver [27], and possibly account for the persistence of effects observed in chronic T1AM administration [3] (Figure 2).

\section{Biological Effects}

The most striking characteristics of T1AM are its radically divergent effects from that of its structural analog, thyroid hormone. In contrast to TH's long term sustained effects on increasing metabolic rate through activation of TR $\alpha$ and TR $\beta$ transcription factors to induce gene expression, administration of T1AM is able to induce a hypometabolic response in minutes [1]. Primary among these hypometabolic effects is its rapid induction of hypothermia $[1,6]$. In the initial study, Scanlan et al. [1] found that when administered to mice via intraperitoneal injection at dosages of $25-100 \mathrm{mg} / \mathrm{kg}$, T1 AM was able to result in an approximate $4^{\circ} \mathrm{C}$ drop in rectal temperature within 30 minutes [1]. At dosages of 50 and $100 \mathrm{mg} / \mathrm{kg}$ this drop in temperature continued to nearly $10^{\circ} \mathrm{C}$ total, with the $100 \mathrm{mg} / \mathrm{kg}$ dosage ultimately proving lethal. From this observation, an $\mathrm{ED}_{50}$ value of T1AM was extrapolated to be $59 \mathrm{mg} / \mathrm{kg}$ in mice. Additional tests revealed that T1AM was able to induce bradycardia and reduced cardiac output as well. These initial observations of T1AMs potent hypometabolic effects have ignited a great deal of interests to apply T1AM as a therapeutic application in a broad number of biological systems.

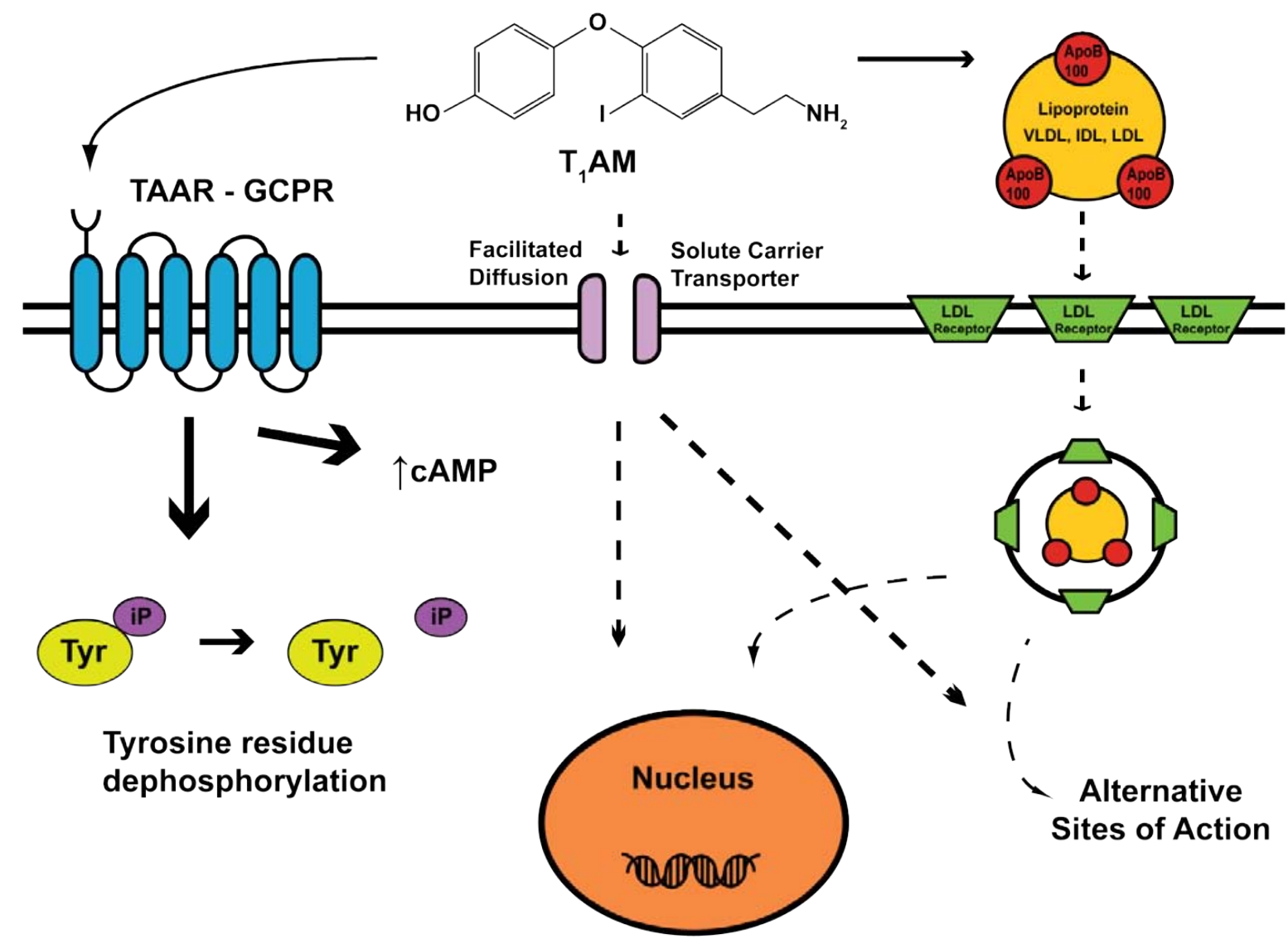

Figure 2: Cellular signaling interactions of T1AM. T1AM acts as a high affinity substrate for trace amine activated receptors, a subfamily of Gcoupled protein receptors. This receptor activation has been shown to enhance tyrosine residue dephosphorylation in vivo and to increase cyclic AMP concentrations in vitro. In addition to cell membrane receptor activation, T1AM can be internalized into the cell. This is likely mediated via facilitated diffusion via a yet be identified solute carrier transporters or possibly by internalization via LDL receptor due to T1AM being bound to ApoB100 containing lipoproteins in circulation. The specific targets of T1AM inside the cell however have yet to be elucidated in the literature. (Solid lines indicate processes substantiated by studies in literature, dashed lines indicate speculated pathways yet to be verified.) 


\section{Brain}

T1AM was initially endogenously detected in rat brain [1]. Due to its structural relation to dopamine and catecholamines and the homology of those compounds' target receptors to TAARs, T1AM was speculated to have neuromodulating properties [7]. T1AM does indeed mediate physiological changes through interactions in the brain and neural tissue, as studies have demonstrated significant responses to T1AM administration in mice utilizing only minute quantities on the order of $\mu \mathrm{g} / \mathrm{kg}$ delivered via intracerebroventricular (ICV) injection [28-30]. Direct microinjection of T1AM into the noradrenergic control region of the brain in mice resulted in increased neuron firing [7]. This response was thought to be mediated through $\alpha 2 \mathrm{~A}$ adrenergic receptor interaction, whereby T1AM possesses adrenergicblocking properties [31]. This interaction with $\alpha 2 \mathrm{~A}$ may present an additional ubiquitous receptor target of T1AM than just GCPRs as it has also been speculated that T1AM interacts with $\alpha 2 \mathrm{~A}$ in pancreatic beta-cells [27]. ICV administration of T1AM was able to improve learning capacity and served as a memory enhancer in mice [28]. Interestingly, ICV administrated T1AM also produced a hyperalgesic effect. These responses are thought to be modulated through interaction with T1AM, or its deaminated derivatives such as $\mathrm{TA}$, on rapid desensitizing targets. The response to T1AM in the brain appears to be quite rapid as the increased concentration of T1AM immediately following ICV injection is largely cleared out within 30 minutes, either by degradation or diversion to the systemic circulation [28]. Indeed the interaction of T1AM in the brain appears to be quite potent, as these micro amounts in the brain are capable of producing systemic physiologic effects, such as hyperglycemia and hypophagia [2830]. These observations suggest that interaction of T1AM with brain and neural tissue is an important aspect of its systemic response across multiple organ systems in vivo.

In addition to its neuromodulating effects, T1AM demonstrates anti-ischemic properties in the brain, as observed in a focal ischemia stroke model in mice [2]. Interestingly, this abatement of ischemic damage was incurred from both preinfarction conditioning and post-infarction administration via IP injection. However, this positive effect was ablated if the hypothermic response due to T1AM administration was blocked. This finding presents an exciting potential application of T1AM as an anti-ischemic treatment for both acute infarction and prevention of ischemic stroke.

\section{Cardiovascular}

Since its initial discovery, cardiovascular effects of T1AM have been documented [1]. T1AM administration in mice was able to induce bradycardia, and acted as a negative inotropic agent that decreased cardiac output as well as systolic blood pressure. These effects may be in part due to interaction or opposition to cellular adrenergic signaling as the drop in systolic blood pressure measured in perfused rat heart was ablated with administration of isoproterenol, a $\beta 2$ agonist [1]. Later studies have also confirmed the negative inotropic properties of T1AM $[20,32]$. Using electrophysiological measurements, one study was able to conclude that T1AM's negative inotropic effects are due to alterations in calcium and potassium flux in cardiomyocytes mediated through increased diastolic leak in sarcoplasmic reserves of calcium as well as increased action potential duration [32]. This negative inotropic effect of T1AM is dose dependent, with increasing dosages resulting in greater decrease in cardiac output $[1,20]$. These negative chronotropic and inotropic effects in the heart make T1AM a promising candidate for reducing ischemic injury due to myocardial infarction. Using a perfused rat heart model, T1AM was demonstrated to help prevent tissue damage from ischemia-reperfusion injury [33]. This ability to prevent ischemic injury was dose dependent in a bell shaped curve pattern with peak effect at $1.25 \mu \mathrm{M}$. The capacity of T1AM to prevent ischemic injury is modulated in part by Protein Kinase C (PKC), as its inhibitor chelerythrine was able to negate the cardioprotective effect of T1AM. T1AM's capacity to prevent ischemic injury was also countered by glibenclamide, a $\mathrm{K}^{+} \mathrm{ATP}$ channel blocker, effectively disrupting mitochondrial permeability transition, a proposed aspect of ischemiareperfusion injury [33]. In addition, the chronic application of T1AM at 10 and $25 \mathrm{mg} / \mathrm{Kg}$ shows heart protection against oxidative stress [4]. Coupled with the rapid onset of T1AMs effects, its negative inotropic properties may prove an effective acute treatment at the onset of a myocardial infarction to prevent the development of irreversible ischemic damage.

\section{Sleep and torpidity}

Due to T1AM's potent hypothermic effects, it is easy to postulate a physiological role in modulating sleep patterns as well as torpidity in hibernating vertebrates. Lowered body temperature is characteristic of the torpid state in mammals. Using a mouse model, a non-hibernating rodent, Ju et al. [34] were able to produce sustained cycle of torpidity over the course of 10 days using a 2 day torpor 1 day arousal pattern to mimic natural hibernation patterns with T1AM administration. This torpid state was sustained with repeated injections of T1AM at $50 \mathrm{mg} / \mathrm{kg}$ when body temperature recovered to $32^{\circ} \mathrm{C}$. No current research has examined specifically the role of T1AM in naturally hibernating species or if it is necessary for the torpor state in these animals. This is a promising avenue of research as induction of a torpid state in mammals has promising applications for emergency situations when urgent care facilities are not immediately available as well as future long term space travel. Given its hypometabolic effects and ability to induce torpor in mice, T1AM would be a likely candidate as an acute acting sleep aid. However, a study utilizing an injection of T1AM into the pre-optic area of the brain in mice found that T1AM reduced EEG-defined sleep in a dose pattern similar to T3 injection [6]. This may represent a divergent response from natural T1AM function due to the route of administration, as other studies have demonstrated a decrease in activity in response to IP administration of T1AM $[1,34]$ or no change $[3,35]$ in contrast to elevated activity levels observed following preoptic injection [6]. Future studies are needed to elucidate the effect on the route of administration of T1AM and identifying the target systems that modulate T1AM's influence on the activity and arousal level. 


\section{Lipid oxidation and glucose homeostasis}

Given the hypometabolic response of T1AM administration, it is a natural hypothesis to suspect that it can modulate changes in macronutrient utilization. Acute IP injection of T1AM $(50 \mathrm{mg} / \mathrm{kg})$ resulted in decreased oxygen consumption $\left(\mathrm{VO}_{2}\right)$ and Respiratory Quotient (RQ), indicating a shift towards lipid utilization, in both Djungarian hamsters and C57BL/6 mice [36]. T1AM appears to deter glucose utilization as this fuel shift was also accompanied by ketonuria. RQ changes were quite drastic, shifting from $\sim 0.91$ in hamsters to $\sim 0.77$ and 0.83 in mice to 0.71 , representing a near complete shift to fat utilization. Most interesting however is that this shift in RQ was observed to last several hours, in contrast to the hypothermic effect of T1AM administration that has previously been observed to subside within 4 hours [1]. Despite a hypometabolic effect, the increased fat utilization produced significant weight loss in the rodents at 24 hours following T1AM injection, with significant decrease in fat mass, but not lean mass. Expanding upon these initial observations regarding macronutrient fuel utilization, a later study demonstrated that T1AM injection of an equivalent dose in rats increases endogenous glucose production and subsequent plasma glucose levels, as well as elevations in plasma glucagon and corticosterone [30]. Equally fascinating, these shifts in glucose metabolism markers were reproduced in an equally potent manner with ICV injection at $\sim 1 \%$ of the IP dosage, giving further credence to the importance of the brain as a target organ modulating the metabolic response to T1AM. Unexpectedly, these elevated glucose levels did not incur an insulin response. Increased hepatic glucose production and blunted insulin secretion suggest a similar interaction in both the liver and pancreatic tissue. This mimics the pattern of adrenergic receptor stimulation in those tissues by which T1AM may initiate through its target receptor TAARs which share considerable homology with those receptors.

This modulation of lipid metabolism has great potential in therapeutic applications for weight loss and metabolic dysfunction. To date the majority of studies utilizing exogenous T1AM have utilized acute supra-physiological boluses of $50 \mathrm{mg} /$ $\mathrm{kg}$, but the metabolic effects on fuel utilization remain intact, albeit less severe, in sustained smaller dosages [3]. Haviland et al. [3] administered T1AM at $10 \mathrm{mg} / \mathrm{kg} /$ day over the course of 8 days in diet-induced obese wild type mice (CD1), which resulted in shift towards fat utilization without the other side effects of hypothermia and hypokinesia observed in higher dosages [1,34]. The shift towards increase lipid utilization was verified by a rapid decrease carbon fractionation in ${ }^{13} \mathrm{CO}_{2} /{ }^{12} \mathrm{CO}_{2}\left(\delta^{13} \mathrm{C}\right.$ values $)$ in breath detected by cavity ring down spectroscopy [37] and increased circulating levels of lipid metabolite intermediate 3-Hydroxybutyrate [3]. This eight-day treatment resulted in significant weight loss at $\sim 8.3 \%$ bodyweight by day 9 , while the mice only regained $1.8 \%$ of the weight loss up to 2 weeks after cessation of the treatment; this is a significant finding as it suggests T1AM administered at physiological dosages can produce sustained weight loss. This long term effect on lipid metabolism may also be mediated by alterations in lipid synthesis as circulating levels of labeled ${ }^{13} \mathrm{C}$-acetate trended towards elevation for 7 days post treatment in response to ${ }^{13} \mathrm{C}$-labeled glucose injection compared to sham controls. This is further supported by observed gene expression changes resulting in upregulation lipolysis and beta-oxidation markers coupled with decreased expression of adipogenesis markers using chronic T1AM treatment ( $10 \mathrm{mg} / \mathrm{kg}$ twice a day for 5 days) in a rat model [27]. In addition to, and perhaps coupled with the increased lipolysis, ketone body metabolism is altered in response to T1AM treatment whereby studies noted ketonuria in response to acute T1AM administration [36], along with low dose chronic T1AM administration resulting in elevation of protein breakdown after 5 days of treatment [3]. This is a potentially adverse response and undesirable aspect of total weight loss, but can perhaps be attenuated through further fine tuning of dosage pattern and dosage amount as the onset of this effect was considerably delayed compared to the acute high dose administration.

These changes to lipid and glucose metabolism are also likely to be mediated through changes in gene expression. Chronic T1AM administration (over 7 days) in female CD-1 mice resulted in upregulation of Sirtuin 6 (SIRT6) and Glucokinase (GK) with down regulation of SIRT4, with elevations of SIRT6 in adipose tissue [38]. This sheds light on T1AM's broad modulation of lipid and glucose metabolism as SIRT6 is a gene regulator of glucose levels and SIRT4 acts as a negative regulator of fatty acid oxidation [38]. This is supported by gene expression assessment in male Windsor rats in response to 5 day treatment of T1AM that showed modulation of gene expression related to lipolysis in and beta-oxidation [27]. In adipose tissue, peroxisomal biogenesis factor 5 (Pex5) and acyl-CoA synthetase long-chain family member 5 (Acsl5) were upregulated while in the liver glycerol kinase and malic enzyme 1 (NADP (+) dependent cytosolic) were down regulated. Taken together these findings provide a basis for attributing T1AM's increased lypolytic and decreased lypogenic metabolic effects in part to alternate gene expression in response to subchronic T1AM treatment. Further examination is needed to understand the effect of T1AM on gene expression in other organ systems and if these patterns diverge in a dose-dependent or frequency dependent manner as well.

\section{Mitochondria and cellular redox}

Though many effects of T1AM appear to be absent in lower dosages [1] the divergent response to T1AM across dosage levels presents further means of inquiry about T1AM metabolic control [3]. This is underscored by a recent study examining mitochondria effects of T1AM whereby low physiologic levels in cardiomyocytes enhanced ADP-stimulated mitochondrial respiration while higher levels inhibited $\mathrm{F}_{0} \mathrm{~F}_{1}$-ATP synthase activity [39]. T1AM is also observed to interact with Complex III via antimycin $\mathrm{A}$ in mitochondria, decreasing $\mathrm{O}_{2}$ consumption and increasing $\mathrm{H}_{2} \mathrm{O}_{2}$ release in mitochondria of cultured hepatocytes, which may partially explain observed decrease in $\mathrm{O}_{2}$ consumption in vivo [39]. Most recently, Ghanian et al. [4] found that T1AM is able to modulate cellular redox state (NADH/FAD ratio) in mouse tissue-specifically and in a dose specific manner [4]; interestingly this effect was divergent between dosage and tissue type. High dosage $(25 \mathrm{mg} / \mathrm{kg})$ induced oxidative stress 
Table 1: Summary of Biological Effects of T1AM.

\begin{tabular}{|c|c|c|c|}
\hline Target Organ/System & Effect & Dosage & Reference \\
\hline Brain & $\uparrow$ neuron fire rate, $\uparrow$ learning, $\uparrow$ memory, hyperalgesic, anti-ischemic & Acute & $2,7,28$ \\
\hline Heart & $\downarrow$ heart rate, $\downarrow$ contractility, anti-ischemic & Acute & $1,19,32,33$ \\
\hline \multirow{2}{*}{ Circadian Clock } & $\downarrow$ EEG-defined sleep & Acute & 6 \\
\hline & Induction of torpidity & Chronic & 34 \\
\hline Mitochondria & $\begin{array}{l}\text { } O \text { Organ specific alterations of cellular redox status, } \\
\uparrow \mathrm{F}_{\mathrm{o}} \mathrm{F}_{1} \text {-ATP synthase activity (low concentration) } \downarrow \mathrm{F}_{0} \mathrm{~F}_{1} \text {-ATP synthase activity (high concentration) }\end{array}$ & Acute & 4,39 \\
\hline \multirow{2}{*}{$\begin{array}{l}\text { Macronutrient } \\
\text { Metabolism }\end{array}$} & Tblood glucose, \glycolysis, $\uparrow$ lipolysis & Acute & 36 \\
\hline & †lipolysis, $\downarrow$ lipid synthesis, $\uparrow$ proteolysis & Chronic & 3 \\
\hline \multirow{2}{*}{ Metabolic Rate } & $\downarrow \mathrm{VO}_{2}, \downarrow \downarrow$ core temperature & Acute & $1,6,34,36$ \\
\hline & $\downarrow$ body mass & Chronic & 3 \\
\hline
\end{tabular}

T1AM affects a broad range of physiological processes and organ systems. The physiology of T1AM appears to be complex as its effects at similar dosages can have opposing effects in different organs [4] or opposing effects on the same system at different dosages [39]. The specific physiologic response appears to be further complicated by differing responses to duration and dosage pattern; acute administration of high dosages of T1AM (50 mg/kg) results in rapid induction of hypothermia [1] and a drop in metabolic rate [36], but chronic low dosages of T1AM (10 mg/kg repeated over 8 days) results in elevated lipolysis and sustained reduction in body mass [3]. Differing dosage patterns and amounts appear to have dramatically divergent effects on the same physiologic systems, suggesting that these differing effects may be mediated through separate yet to be identified mechanisms. These multifaceted functions of T1AM provide evidence of its broad spanning importance in human health and potential for therapeutic applications across multiple organ systems. Further research is required in order to titrate proper dosage amount and dosage duration to achieve the desired therapeutic effect in the target physiologic system.

(OS) in kidneys with no elevation in OS in response to low dosage $(10 \mathrm{mg} / \mathrm{kg})$ compared to controls. This was in stark contrast to lowered OS in both high and low dose T1AM treatment in heart tissue compared to control mice. These alternative responses in different tissue to varying dosages of T1AM suggest there are different receptor expression patterns either through different levels of T1AM target receptor expression or differing isoforms resulting in divergent uptake patterns and/or receptor affinity mediating the differing responses between tissues. Furthermore, this ability to alter cellular redox state may shed additional light on T1AM's with more persistent effects, which would likely modulate cellular function internally beyond its acute membrane receptor mediated signaling; NAD is the substrate required for the function of Sirtuins, a family of enzymes that regulate epigenetic deacetylation and protein ribosalation influencing gene transcription and mitochondrial function (AssadiPorter, unpublished results) $[4,38]$. T1AM's role in modulating mitochondrial function, with sirtuins being a promising mediator, is the area of research that may be a key to unlock the specific mechanisms by which T1AM is able to alter whole body and cellular metabolism.

\section{Summary}

Since T1AM's initial discovery as a new thyroid hormone analog, new areas of research have opened with it, along with very promising potential for therapeutic uses in a wide array of physiologic systems touched on in this review: sleep, torpor, ischemic injury, weight loss, lipid and glucose homeostasis, and cellular redox state (Table 1). The total body of research conducted on T1AM remains relatively small at the time of this review, as it has been only 10 years since its discovery. Many issues in the current body of T1AM literature remain to be resolved where future research should attempt to answer: 1 .
No demonstrable biosynthetic pathway responsible for T1AM formation or identity of the primary synthesizing tissue(s) has been presented. 2 . The specific target tissues and cell types that exert T1AM's systemic effect remains to be definitively identified. 3. The specific mechanisms causing divergent responses to differing dosages across different tissues have yet to be explained. 4. The specific cellular mechanisms and transporters responsible for cellular uptake of T1AM are not decisively known. 5. The importance of T1AM in the physiology of hibernating animals remains to be examined. 6 . No relationship has been established either though negative feedback inhibition, thyroid hormones, or competing trace amines that regulates its endogenous secretion. 7. Once taken into the cell, the specific intracellular targets are virtually a complete mystery. Given its promising therapeutic potential, extensive further study is warranted on this new hormone metabolite. By harnessing the hypometabolic and lipid oxidizing properties of T1AM for therapeutic applications, better understanding of T1AM has greater promise for advancing the science of human health.

\section{References}

1. Scanlan TS, Suchland KL, Hart ME, Chiellini G, Huang Y, Kruzich PJ, et al. 3-Iodothyronamine is an endogenous and rapid-acting derivative of thyroid hormone. Nat Med. 2004; 10(6):638-642.

2. Doyle KP, Suchland KL, Ciesielski TM, Lessov NS, Grandy DK, Scanlan TS, et al. Novel thyroxine derivatives, thyronamine and 3-iodothyronamine, induce transient hypothermia and marked neuroprotection against stroke injury. Stroke. 2007; 38(9):25692576.

3. Haviland JA, Reiland H, Butz DE, Tonelli M, Porter WP, Zucchi R, et al. NMR-based metabolomics and breath studies show lipid and protein catabolism during low dose chronic T1AM treatment. Obesity (Silver Spring). 2013; 21(12):2538-2544. doi: 10.1002/oby.20391. 
4. Ghanian Z, Maleki S, Reiland H, Butz DE, Chiellini G, Assadi-Porter, et al. Optical imaging of mitochondrial redox state in rodent models with 3-iodothyronamine. Exp Biol Med (Maywood). 2014; 239(2):151-158. doi: $10.1177 / 1535370213510252$.

5. Zucchi R, Ghelardoni S, Chiellini G. Cardiac effects of thyronamines. Heart Fail Rev. 2010; 15(2):171-176. doi: 10.1007/s10741-0089120-z.

6. James TD, Moffett SX, Scanlan TS, Martin JV. Effects of acute microinjections of the thyroid hormone derivative 3-iodothyronamine to the preoptic region of adult male rats on sleep, thermoregulation and motor activity. Horm Behav. 2013; 64(1):81-88. doi: 10.1016/j. yhbeh.2013.05.004.

7. Gompf HS, Greenberg JH, Aston-Jones G, Ianculescu AG, Scanlan TS, Dratman MB. 3-Monoiodothyronamine: the rationale for its action as an endogenous adrenergic-blocking neuromodulator. Brain Res. 2010; 1351:130-140. doi: 10.1016/j.brainres.2010.06.067.

8. Lee JS, Jang BS, Chung CM, Choi I, Kim JG, Park SH. In vivo molecular imaging of [125I]-labeled 3-iodothyronamine: a hibernationinducing agent. Appl Radiat Isot. 2013; 73:74-78. doi: 10.1016/j. apradiso.2012.11.017.

9. Scanlan TS. Minireview: 3-Iodothyronamine (T1AM): a new player on the thyroid endocrine team? Endocrinology. 2009; 150(3):11081111. doi: 10.1210/en.2008-1596.

10. Wu SY, Green WL, Huang WS, Hays MT, Chopra IJ. Alternate pathways of thyroid hormone metabolism. Thyroid. 2005; 15(8):943-958.

11. Piehl S, Heberer T, Balizs G, Scanlan TS, Smits R, Koksch B, et al. Thyronamines are isozyme-specific substrates of deiodinases. Endocrinology. 2008; 149(6):3037-3045. doi: 10.1210/en.20071678.

12. Chopra IJ. An assessment of daily production and significance of thyroidal secretion of 3, 3', 5' -triiodothyronine (reverse T3) in man. J Clin Invest. 1976; 58(1):32-40.

13. Hackenmueller SA, Marchini M, Saba A, Zucchi R, Scanlan TS Biosynthesis of 3-iodothyronamine (T1AM) is dependent on the sodium-iodide symporter and thyroperoxidase but does not involve extrathyroidal metabolism of T4. Endocrinology. 2012; 153(11):56595667. doi: 10.1210/en.2012-1254.

14. Hoefig CS, Kohrle J, Brabant G, Dixit K, Yap B, Strasburger CJ, et al. Evidence for extrathyroidal formation of 3-iodothyronamine in humans as provided by a novel monoclonal antibody-based chemiluminescent serum immunoassay. J Clin Endocrinol Metab. 2011; 96(6):1864-1872. doi: 10.1210/jc.2010-2680.

15. Saba A, Chiellini G, Frascarelli S, Marchini M, Ghelardoni S, Raffaelli A, et al. Tissue distribution and cardiac metabolism of 3-iodothyronamine. Endocrinology. 2010; 151(10):5063-5073. doi: 10.1210/en.20100491.

16. Pietsch CA, Scanlan TS, Anderson RJ. Thyronamines are substrates for human liver sulfotransferases. Endocrinology. 2007; 148(4):19211927.

17. Wood WJ, Geraci T, Nilsen A, DeBarber AE, Scanlan TS Iodothyronamines are oxidatively deaminated to iodothyroacetic acids in vivo. Chembiochem. 2009; 10(2):361-365. doi: 10.1002/ cbic. 200800607.

18. Borowsky B, Adham N, Jones KA, Raddatz R, Artymyshyn R, Ogozalek $\mathrm{KL}$, et al. Trace amines: identification of a family of mammalian G protein-coupled receptors. Proc Natl Acad Sci U S A. 2001; 98(16):8966-8971.
19. Panas HN, Lynch LJ, Vallender EJ, Xie Z, Chen GL, Lynn SK, et al. Normal thermoregulatory responses to 3-iodothyronamine, trace amines and amphetamine-like psychostimulants in trace amine associated receptor 1 knockout mice. J Neurosci Res. 2010; 88(9):1962-1969. doi: 10.1002/jnr.22367.

20. Chiellini G, Frascarelli S, Ghelardoni S, Carnicelli V, Tobias SC, DeBarber A, et al. Cardiac effects of 3-iodothyronamine: a new aminergic system modulating cardiac function. FASEB J. 2007; 21(7):1597-1608.

21. Frascarelli S, Ghelardoni S, Chiellini G, Vargiu R, Ronca-Testoni S, Scanlan TS, et al. Cardiac effects of trace amines: pharmacological characterization of trace amine-associated receptors. Eur J Pharmacol. 2008; 587(1-3):231-236. doi: 10.1016/j.ejphar.2008.03.055

22. Hart ME, Suchland KL, Miyakawa M, Bunzow JR, Grandy DK, Scanlan TS. Trace amine-associated receptor agonists: synthesis and evaluation of thyronamines and related analogues. J Med Chem. 2006; 49(3):1101-1112.

23. Chiellini G, Erba P, Carnicelli V, Manfredi C, Frascarelli S, Ghelardoni S, et al. Distribution of exogenous [125I]-3-iodothyronamine in mouse in vivo: relationship with trace amine-associated receptors. J Endocrinol. 2012; 213(3):223-230. doi: 10.1530/JOE-12-0055.

24. Muhlhaus J, Dinter J, Nurnberg D, Rehders M, Depke M, Golchert J, et al. Analysis of human TAAR8 and murine Taar8b mediated signaling pathways and expression profile. Int J Mol Sci. 2014; 15(11):2063820655. doi: 10.3390/ijms151120638.

25. Roy G, Placzek E, Scanlan TS. ApoB-100-containing lipoproteins are major carriers of 3-iodothyronamine in circulation. J Biol Chem. 2012; 287(3):1790-1800. doi: 10.1074/jbc.M111.275552.

26. Ianculescu AG, Giacomini KM, Scanlan TS. Identification and characterization of 3-iodothyronamine intracellular transport. Endocrinology. 2009; 150(4):1991-1999. doi: 10.1210/en.20081339.

27. Mariotti V, Melissari E, Iofrida C, Righi M, Di Russo M, Donzelli R, et al. Modulation of gene expression by 3-iodothyronamine: genetic evidence for a lipolytic pattern. PLoS One. 2014; 9 (11): e106923. doi: 10.1371/journal.pone.0106923.

28. Manni ME, De Siena G, Saba A, Marchini M, Landucci E, Gerace E, et al. Pharmacological effects of 3-iodothyronamine (T1AM) in mice include facilitation of memory acquisition and retention and reduction of pain threshold. Br J Pharmacol. 2013; 168(2):354-362. doi: 10.1111/j.1476-5381.2012.02137.x.

29. Manni ME, De Siena G, Saba A, Marchini M, Dicembrini I, Bigagli E, et al. 3-Iodothyronamine: a modulator of the hypothalamus-pancreasthyroid axes in mice. Br J Pharmacol. 2012; 166(2):650-658. doi: 10.1111/j.1476-5381.2011.01823.x.

30. Klieverik LP, Foppen E, Ackermans MT, Serlie MJ, Sauerwein HP, Scanlan TS, et al. Central effects of thyronamines on glucose metabolism in rats. J Endocrinol. 2009; 201(3):377-386. doi: 10.1677/ JOE-09-0043.

31. Regard JB, Kataoka H, Cano DA, Camerer E, Yin L, Zheng YW, et al. Probing cell type-specific functions of $\mathrm{Gi}$ in vivo identifies GPCR regulators of insulin secretion. J Clin Invest. 2007; 117(12):40344043.

32. Ghelardoni S, Suffredini S, Frascarelli S, Brogioni S, Chiellini G, Ronca-Testoni S, et al. Modulation of cardiac ionic homeostasis by 3-iodothyronamine. J Cell Mol Med. 2009; 13(9B):3082-3090. doi: 10.1111/j.1582-4934.2009.00728.x.

33. Frascarelli S, Ghelardoni S, Chiellini G, Galli E, Ronca F, Scanlan TS, et 
al. Cardioprotective effect of 3-iodothyronamine in perfused rat heart subjected to ischemia and reperfusion. Cardiovasc Drugs Ther. 2011; 25(4):307-313. doi: 10.1007/s10557-011-6320-x.

34.Ju H, So H, Ha K, Park K, Lee JW, Chung CM, et al. Sustained torpidity following multi-dose administration of 3-iodothyronamine in mice. J Cell Physiol. 2011; 226(4):853-858. doi: 10.1002/jcp.22573.

35. Dhillo WS, Bewick GA, White NE, Gardiner JV, Thompson EL, Bataveljic A, et al. The thyroid hormone derivative 3-iodothyronamine increases food intake in rodents. Diabetes Obes Metab. 2009; 11(3):251-260. doi: 10.1111/j.1463-1326.2008.00935.x.

36. Braulke LJ, Klingenspor M, DeBarber A, Tobias SC, Grandy DK, Scanlan TS, et al. 3-Iodothyronamine: a novel hormone controlling the balance between glucose and lipid utilisation. J Comp Physiol B. 2008; 178(2):167-177.
37. Haviland JA, Tonelli M, Haughey DT, Porter WP, Assadi-Porter FM. Novel diagnostics of metabolic dysfunction detected in breath and plasma by selective isotope-assisted labeling. Metabolism. 2012; 61(8):1162-1170. doi: 10.1016/j.metabol.2011.12.010.

38. Assadi-Porter FM, Tonelli M, Chiellini G. A novel role of 3-iodothyronamine (T1AM) as a master regulator of lipid and glucose metabolism through activating sirtuins 4 and 6 genes. FASEB J. 2014; 28(1): Supplement 373.7.

39. Cumero S, Fogolari F, Domenis R, Zucchi R, Mavelli I, Contessi S. Mitochondrial $\mathrm{F}_{0} \mathrm{~F}_{1}$-ATP synthase is a molecular target of 3 -iodothyronamine, an endogenous metabolite of thyroid hormone. Br J Pharmacol. 2012; 166 (8): 2331-2347. doi: 10.1111/j.14765381.2012.01958.x 